\title{
Reversible Topochemical Polymerization and Depolymerization of a Crystalline Three-Dimensional Porous Organic Polymer with $\mathrm{C}-\mathrm{C}$ Bond Linkages
}

Chenyue Sun, Julius J. Oppenheim, Grigorii Skorupskii, Luming Yang, Mircea Dincă*

Department of Chemistry, Massachusetts Institute of Technology, Cambridge, Massachusetts 02139, United

States

Email: mdinca@mit.edu

\begin{abstract}
Three-dimensionally connected porous organic polymers are of interest because of their potential in adsorption, separation, and sensing, among others. When crystalline, they also afford accurate structure description, which in turn can enable particular functions. However, crystallization of three-dimensional (3D) polymers is challenging. This is especially true when targeting polymerization via stable $\mathrm{C}-\mathrm{C}$ bonds, whose formation is usually irreversible and does not allow for error correction typically required for crystallization. Here, we report polyMTBA, the first 3D-connected crystalline organic polymer with permanent porosity, here formed via $\mathrm{C}-\mathrm{C}$ linkages. High crystallinity is achieved by solid-state topochemical reaction within monomer MTBA crystals. polyMTBA is recyclable via thermal depolymerization and is solution-processable via its soluble monomers. These results reveal topochemical polymerization as a compelling methodology for generating stable, crystalline, and porous 3D organic frameworks.
\end{abstract}

\section{Main}

Organic porous materials such as hypercrosslinked polymers, ${ }^{1}$ covalent organic frameworks (COFs) ${ }^{2}$ conjugated microporous polymers (CMPs), ${ }^{3}$ and porous aromatic frameworks (PAFs) ${ }^{4}$ have garnered considerable attention for their utility in adsorption, ${ }^{5-9}$ catalysis, ${ }^{10-12}$ and energy storage. ${ }^{13-18}$ Among these, COFs stand out because of their crystallinity, which both allows for accurate description and understanding of their structure, and confers unique benefits such as monodisperse size-selective sieving, shape selection, and control over capillary condensation. ${ }^{19-21}$ On the other hand, CMPs, PAFs, and hypercrosslinked polymers stand out through their typically enhanced chemical stability afforded by irreversible $\mathrm{C}-\mathrm{C}$ and aromatic linkages. ${ }^{22-25}$ Despite recent advances in $\mathrm{C}=\mathrm{C}$-linked two-dimensional (2D) $\mathrm{COFs},{ }^{26}$ it is still challenging to form crystalline $\mathrm{C}-\mathrm{C}$-linked porous frameworks via solvothermal routes because these require irreversible bond formation and crystallization in a single step, which is incompatible with error correction during crystal growth.

To this end, topochemical polymerization provides a promising avenue to stable, highly crystalline materials. ${ }^{27}$ With topochemical polymerization, monomer polymorphs are chosen in such a way that the reactive functional groups are spatially aligned in close proximity to allow for bond formation in the solid state. Subsequent $\mathrm{C}-\mathrm{C}$ bond formation, triggered by light or some other stimuli, is effectively decoupled from crystallization and thus gives rise to highly crystalline polymers. Although early examples of topochemical polymerization primarily focused on generating one-dimensional polymers, particularly with diynes and dienes, ${ }^{27,28}$ in recent years, increasing efforts have been devoted to $2 \mathrm{D}$ polymers, taking advantage of the photochemical dimerization reaction of stilbene and anthracene derivatives. ${ }^{29-33}$ To our knowledge, however, this methodology has not yet been extended to produce $3 \mathrm{D}$ crystalline organic polymers. 
Here, we report that photochemical polymerization of methanetetrayltetrakis(benzene-4,1-diyl) tetrakis(anthracene-9-carboxylate) (MTBA, Fig 1a) crystals in the solid state yields a crystalline porous solid (polyMTBA, Fig. 1f) with permanent porosity. Even though MTBA crystals have no discernible crystallographic voids, porosity emerges in polyMTBA due to a large displacement of monomer molecules during polymerization, which creates one-dimensional channels along the $c$-direction (Fig 1e-f). polyMTBA can be deposited onto glass substrates from solution-processed MTBA films, and can be thermally degraded to recover pristine monomer.

The molecular structure of MTBA was carefully chosen to favor a rigid and open framework structure. Anthracene is attractive as a topochemical motif because of its relatively small size and its well-documented photodimerization chemistry. ${ }^{34}$ In particular, 9-substituted anthracenes tend to crystallize and dimerize in headto-tail fashion (Fig. 1b). Inspiration for the tetrahedral tetraphenylmethane core in MTBA came from COFs, ${ }^{35,36}$ which use this motif to impose high rigidity and maximal angular separation between the peripheral groups, which are conducive to permanent porosity. It should be noted that due to the highly congested nature around the bridgehead carbon atoms in dianthracene, the range of permissible substituents at the 9 and 10 position of anthracene is quite limited. For instance, 9,10-diphenylanthracene is photochemically inert in both solution and solid state, likely because the ortho-H on the phenyl group prevents the proximity of two anthracene units in the transition state. ${ }^{37}$ A sterically less demanding ester linkage, on the other hand, does not affect the dimerization. ${ }^{38}$

MTBA crystallizes in the tetragonal space group $\mathrm{I}_{1} / \mathrm{a}$ with no lattice solvent. Individual MTBA molecules stack into columns along the $c$-direction, collinear with the $S_{4}$ molecular axis (Fig. 1d). Each anthracene group is paired face-to-face (dihedral angle $<0.01^{\circ}$, interplanar distance $3.3-3.6 \AA$ ) with an anthracene from a neighboring molecule. However, significant slipping displacement between the two neighboring anthracene groups gives rise to a large C9 'C10' separation of 5.3-5.6 $\AA$ (Supplementary Fig. 1), which is much larger than what is commonly believed to be the maximal separation ( $4.2 \AA$, Schmidt criterion ${ }^{39}$ ) allowed for topochemical anthracene dimerization. However, the two neighboring anthracene groups still interact electronically and give rise to excimeric emission, as exhibited by the green photoluminescence $\left(\lambda_{\max }=503 \mathrm{~nm}\right.$, Supplementary Fig. 2$)$ of a polycrystalline powder of MBTA. 


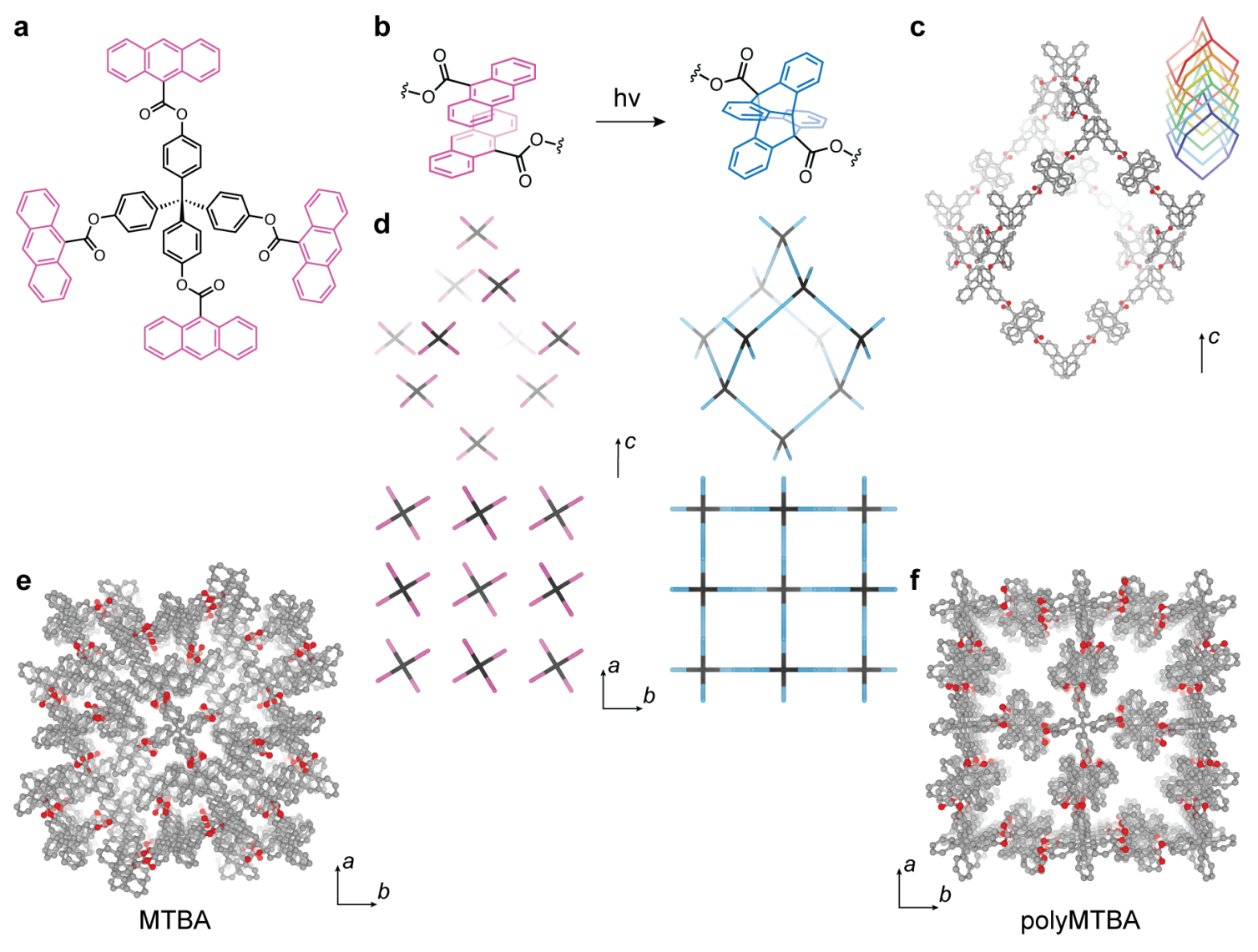

Fig. 1. Structural details of MTBA and polyMTBA. a, the molecular structure of monomer MTBA. $\mathbf{b}$, adjacent anthroate pairs in crystalline MTBA undergo dimerization upon irradiation. $\mathbf{c}$, A single adamantane-like cage in polyMTBA. Actual polyMTBA is 6-fold interpenetrated (inset). d, Individual MTBA building units are prearranged in the crystalline phase (left); these units are then covalently linked to form the diamond-type framework in polyMTBA (right). A single isolated network is shown for clarity. $\mathbf{e}$ and $\mathbf{f}$, Representations of the crystal structures of MTBA (e, single crystal X-ray diffraction data) and polyMTBA (f, molecular mechanics model based on Pawley refinement of unit cell parameters from powder X-ray diffraction). Hydrogen atoms are omitted for clarity.

Despite the large C9 $\cdots$ C10' separation, irradiation of MTBA crystals at $370 \mathrm{~nm}$ or $427 \mathrm{~nm}$ induced a noticeable change of the fluorescence from intense green to faint sky-blue, while the solid turned from bright yellow to beige. In our hands, the most complete transformation, as assessed by adsorption capacity (vide infra), was obtained by irradiating a continuously stirred suspension of powder MTBA in diethyl ether under 1 bar of $\mathrm{CO}_{2}$ with a $427 \mathrm{~nm}$ LED for 3 days. The absence of $\mathrm{CO}_{2}$ during irradiation leads to a product exhibiting lower porosity, leading us to believe that $\mathrm{CO}_{2}$ may fill pores generated during topochemical transformation, thereby compensating for the loss of dispersive interaction between MTBA molecules. Additionally, MTBA itself adsorbs $13.3 \mathrm{~cm}^{3} / \mathrm{g} \mathrm{CO}_{2}$ at 1 bar and $273 \mathrm{~K}$, confirming that $\mathrm{CO}_{2}$ penetrates the monomer lattice to some extent (Supplementary Fig. 3). Importantly, diethyl ether does not dissolve MTBA and therefore serves as a good suspension medium for monomer powder, allowing for better irradiation exposure. Furthermore, $427 \mathrm{~nm}$ is at 
the edge of the absorption peak of MTBA (Supplementary Fig. 4); irradiation at this wavelength improves light penetration into the crystals. ${ }^{40}$
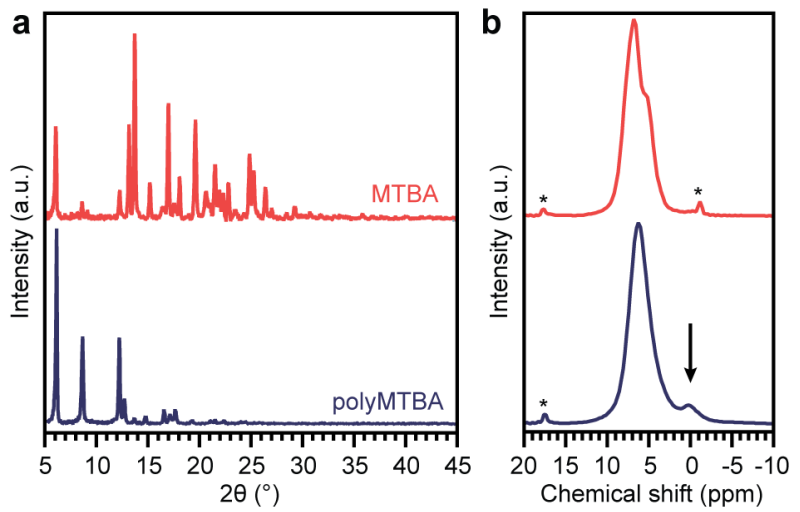

Fig. 2. Qualitative and quantitative evidence for transformation from MTBA to polyMTBA. a, Transformation is evident from significant changes in the intensity and peak positions of the powder X-ray diffraction (PXRD) patterns. b, Quantitative ${ }^{1} \mathrm{H}$ solid-state nuclear magnetic resonance (SS-NMR) spectra show clear appearance of the bridgehead benzylic proton (denoted by an arrow) upfield of the aromatic signals. Strong intermolecular shielding causes large upfield shifts. Asterisks denote artifact signals from sample rotor/probe.

Characterization of the photoproduct suggests the emergence of a significant amount of dianthracene linkages and loss of spectroscopic features for monomeric anthracene groups. Diffuse-reflectance UV-visible spectroscopy shows significant weakening of the characteristic absorption of anthracene above $300 \mathrm{~nm}$ in the photoproduct (Supplementary Fig. 4). By diffuse-reflectance infrared Fourier-transform spectroscopy (Supplementary Fig. 5), MTBA shows no strong features in the $700-600 \mathrm{~cm}^{-1}$ region, whereas the photoproduct displays two strong bands at $690 \mathrm{~cm}^{-1}$ and $636 \mathrm{~cm}^{-1}$, characteristic of anthroate dimers. ${ }^{41} \mathrm{~A}$ cross-polarized magic-angle spinning ${ }^{13} \mathrm{C}$ SS-NMR spectrum of the photoproduct displays new peaks at approximately $50 \mathrm{ppm}$, which are assigned to the bridgehead carbon in dianthracene (Supplementary Fig. 6). Altogether, vibrational, electronic, and NMR spectroscopy along with the loss of excimeric emission confirm the dimerization of a significant portion of neighboring anthracene units in solid MTBA.

To quantify the degree of photopolymerization, we turned to high-resolution solid-state ${ }^{1} \mathrm{H}-\mathrm{NMR}$ spectroscopy with homonuclear dipolar decoupling. ${ }^{42}$ We note that in the solid state, all protons shift significantly upfield relative to the solution spectra due to the strong shielding effect of nearby aromatic rings, ${ }^{43}$ but the benzylic proton of dianthracenes can still be readily discerned from the aromatic protons (Fig. 2b). Peak integration gives a ratio of $12.8: 1$ aromatic to benzylic protons, which equates to approximately $93 \%$ polymerization. This high degree of photodimerization is surprising given that the separation of anthracene units in MTBA seems to violate the Schmidt criterion (vide supra), but is by no means unprecedented and is likely enabled by significant motion of the monomers under irradiation: solid-state photodimerization reactions involving even larger molecular motions have been reported. ${ }^{44,45}$ Indeed, the relatively slow photopolymerization in our system (days, cf. hours in ref. ${ }^{30,31}$ ) is in line with those involving significant molecular displacement. If we assume that all anthracene groups within each monomer engage in photodimerization, the photoproduct is a $3 \mathrm{D}$ polymer network, i.e. polyMTBA (Figs. 1c and 1d). Consistent with this assignment, the photoproduct is insoluble in common organic solvents. 
Crucially, the transformation from MTBA to polyMTBA preserves crystallinity. The powder X-ray diffraction (PXRD) pattern of polyMTBA is clearly distinct from that of MTBA in both peak positions and relative intensities (Fig. 2a, Supplementary Fig. 8). Scanning electron microscopy (SEM) showed that the square prismatic shape of MTBA crystallites persists after polymerization without noticeable deformation (Supplementary Fig. 7). However, efforts to obtain single crystals of polyMTBA were unsuccessful: even when attempting photopolymerization at low temperature (223 K), single crystals of MTBA rapidly degrade to show only polycrystalline diffraction features. However, a combination of Pawley refinement and molecular mechanics optimization provided reliable insight into the structure of polyMTBA (Fig. 1, Supplementary Figs. 8 and 9). Thus, high-resolution synchrotron PXRD data of polyMTBA can be fitted well in the same I4 $/$ /a space group as the monomer, with notable expansion along both $a$ (28.8432(3) $\AA$ vs 28.2999(3) $\AA$ of MTBA) and $c$ (15.9068(2) $\AA$ vs 15.0898(2) $\AA$ of MTBA). Scaling the experimental crystal structure of MBTA accordingly and replacing the anthracene monomer pairs with dianthracenes gave a polymer model used as the input for molecular mechanics optimization. Overall, the computed fully polymerized polyMTBA can be viewed as a sixfold interpenetrated diamond-type framework (Fig. 1c). Importantly, one-dimensional open channels with a diameter of approximately $3.9 \AA$ extend parallel to the $c$-direction (Fig. 1f, Supplementary Fig. 9).
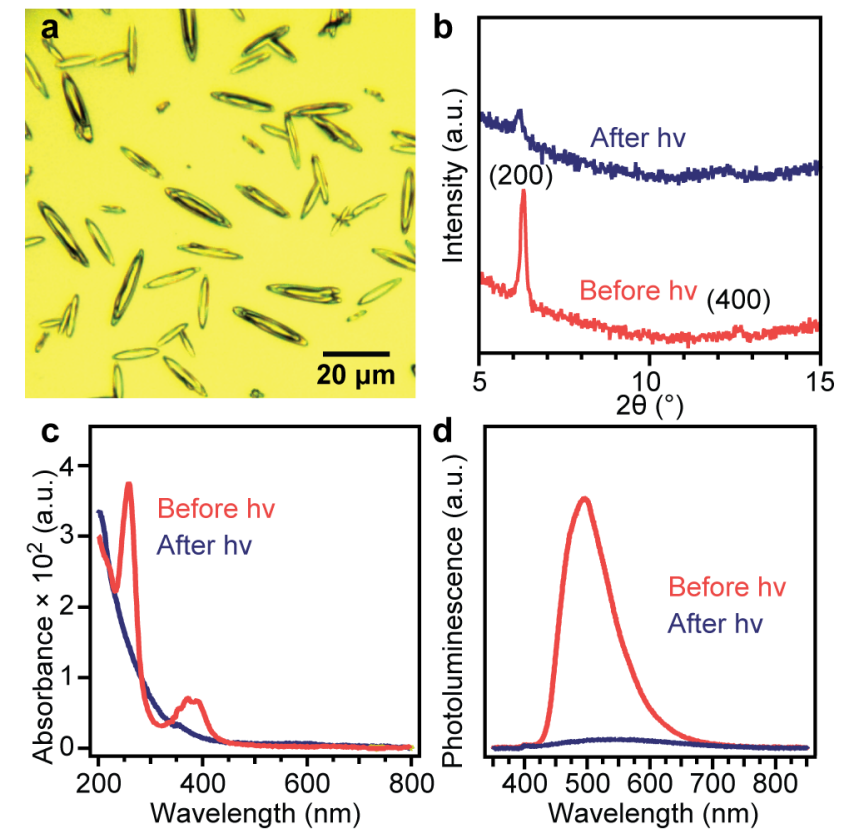

Fig. 3. Characterization of surface-deposited MTBA and polyMTBA. a, Crystallites of polyMTBA on a glass substrate under optical microscope. b. Grazing incidence X-ray diffraction pattern before and after irradiation, showing the (200) diffraction shifting to lower angle. c, UV-visible absorption spectra of MTBA deposited on quartz before and after irradiation. d, Photoluminescence spectra of the same sample as in $\mathbf{c}$.

Thanks to the solubility of MTBA, polyMTBA can be facilely processed into various forms. For instance, spin-coating of an MTBA solution onto glass and quartz substrates forms initially amorphous films, which transform into discrete spindle-shaped crystallites upon solvent-vapor annealing. Subsequent irradiation of this surface-deposited MTBA precursor led to evident shifts in the grazing-incidence X-ray diffraction pattern, indicative of polymerization and formation of polyMTBA (Fig. 3b) without apparent change in the crystallites (Fig. 3a). Surface deposition here allows for efficient light penetration and a high degree of polymerization after 
irradiation (Fig. 3a, Supplementary Fig. 10) as evidenced by the clean disappearance of the anthracene UVvisible absorption above $300 \mathrm{~nm}$ (Fig. 3c) and complete loss of excimeric photoluminescence (Fig. 3d).

$\mathrm{CO}_{2}$ adsorption isotherms confirmed the ultramicroporous nature of polyMTBA. Due to kinetic restrictions, polyMTBA adsorbs minimal $\mathrm{N}_{2}$ at $77 \mathrm{~K} .{ }^{46}$ However, a $\mathrm{CO}_{2}$ isotherm measured at $195 \mathrm{~K}$ exhibits steep uptake at low pressure and a capacity of $108 \mathrm{~cm}^{3} / \mathrm{g} \mathrm{STP}(4.75 \mathrm{mmol} / \mathrm{g}, 14.8 \mathrm{wt} \%)$ at 0.93 bar (Supplementary Fig. 11). At $273 \mathrm{~K}$, polyMTBA adsorbs $31.3 \mathrm{~cm}^{3} / \mathrm{g} \mathrm{STP}(1.38 \mathrm{mmol} / \mathrm{g}, 4.8 \mathrm{wt} \%)$ at 1.0 bar (Supplementary Figs. 11 and 12). This capacity is very close to the value obtained from simulation on the molecular mechanics-optimized model ${ }^{47}\left(34.2 \pm 2.0 \mathrm{~cm}^{3} / \mathrm{g} \mathrm{STP}\right.$ at $\left.1.0 \mathrm{bar}\right)$. Fitting the $\mathrm{CO}_{2}$ isotherm at $195 \mathrm{~K}$ to the BET model gives an apparent surface area of $350 \mathrm{~m}^{2} / \mathrm{g}$ (calc. $408.6 \pm 0.4 \mathrm{~m}^{2} / \mathrm{g}$ for the optimized model).

Importantly, polyMTBA depolymerizes into MTBA at elevated temperature. Thermogravimetric analysis (TGA) of polyMTBA and MTBA under inert atmosphere gives identical results, with a plateau at lower temperature and a rapid mass loss above $375^{\circ} \mathrm{C}$ (Supplementary Fig. 13). The similar thermal behavior between the monomer and the polymer is consistent with the expectation that polyMTBA depolymerizes cleanly below $375{ }^{\circ} \mathrm{C}$. To pinpoint the decomposition temperature, we performed variable-temperature (VT) PXRD of polyMTBA under a helium atmosphere. The characteristic diffraction peaks of the polymer remain largely unchanged below $90^{\circ} \mathrm{C}$, above which diffraction peaks start losing intensity (Supplementary Fig. 14a). At much higher temperature, the residual weak diffraction strongly resembles that of MTBA (Supplementary Fig. 14b). Indeed, an NMR spectrum of the thermolyzed sample is identical to that of pristine MTBA (Supplementary Figs. 15-16). Consistent with the VT-PXRD data, differential scanning calorimetry (DSC) of polyMTBA also reveals exothermic transformations with an onset temperature of approximately $100{ }^{\circ} \mathrm{C}$ (Supplementary Fig. 17), albeit displaying complexities that are in line with the behavior of previously reported 2D topochemical polymers. ${ }^{30}$ Altogether, the TGA, VT-PXRD, and DSC data pin $100{ }^{\circ} \mathrm{C}$ as the onset of thermal depolymerization. Under ambient conditions, minimal depolymerization is observed over weeks (Supplementary Fig. 18).

\section{Conclusions}

The foregoing results describe polyMTBA, a rare example of a 3D-connected, porous crystalline organic polymer. Enabled by topochemical photopolymerization, the formation of polyMTBA as a crystalline product is possible because crystallization of the monomers and the irreversible $\mathrm{C}-\mathrm{C}$ bond formation between the monomers are decoupled. We show that topochemical dimerization of anthracene is possible despite a large C9 $\cdots$ C10' separation of 5.3-5.6 $\AA$, and that one-dimensional channels absent in the monomer crystals are created upon polymerization. The synthetic strategy introduced here enables the material to be recyclable and solutionprocessable, thereby potentially serving as a blueprint for generating other complex and porous frameworks, including by exploiting co-crystallization and co-polymerization of dissimilar monomers.

\section{Data availability}

Experimental and computational details and additional spectroscopic and crystallographic characterization data supporting findings in this paper are available in the Supplementary Information. Crystallographic information for MTBA has been deposited in the Cambridge Crystallographic Data Centre under accession code CCDC 2084357. Computed structure of polyMTBA is also provided as a CIF file. 


\section{References}

1. Davankov, V. A. \& Tsyurupa, M. P. Structure and properties of hypercrosslinked polystyrene-the first representative of a new class of polymer networks. React. Polym. 13, $27-42$ (1990).

2. Côté, A. P. et al. Porous, Crystalline, Covalent Organic Frameworks. Science (80-. ). 310, 1166-1170 (2005).

3. Jiang, J.-X. et al. Conjugated Microporous Poly(aryleneethynylene) Networks. Angew. Chemie Int. Ed. 46, 8574-8578 (2007).

4. Ben, T. et al. Targeted Synthesis of a Porous Aromatic Framework with High Stability and Exceptionally High Surface Area. Angew. Chemie Int. Ed. 48, 9457-9460 (2009).

5. Budd, P. M. et al. Gas separation membranes from polymers of intrinsic microporosity. J. Memb. Sci. 251, 263-269 (2005).

6. Kang, Z. et al. Mixed Matrix Membranes (MMMs) Comprising Exfoliated 2D Covalent Organic Frameworks (COFs) for Efficient CO 2 Separation. Chem. Mater. 28, 1277-1285 (2016).

7. Doonan, C. J., Tranchemontagne, D. J., Glover, T. G., Hunt, J. R. \& Yaghi, O. M. Exceptional ammonia uptake by a covalent organic framework. Nat. Chem. 2, 235-238 (2010).

8. Liang, B. et al. Microporous membranes comprising conjugated polymers with rigid backbones enable ultrafast organic-solvent nanofiltration. Nat. Chem. 10, 961-967 (2018).

9. L Li, B., Zhang, Y., Ma, D., Shi, Z. \& Ma, S. Mercury nano-trap for effective and efficient removal of mercury(II) from aqueous solution. Nat. Commun. 5, 5537 (2014).

10. Kaur, P., Hupp, J. T. \& Nguyen, S. T. Porous Organic Polymers in Catalysis: Opportunities and Challenges. ACS Catal. 1, 819-835 (2011).

11. Xie, Y., Wang, T.-T., Liu, X.-H., Zou, K. \& Deng, W.-Q. Capture and conversion of CO2 at ambient conditions by a conjugated microporous polymer. Nat. Commun. 4, 1960 (2013).

12. Lin, S. et al. Covalent organic frameworks comprising cobalt porphyrins for catalytic $\mathrm{CO} 2$ reduction in water. Science (80-. ). 349, 1208-1213 (2015).

13. Xu, F. et al. Redox-active conjugated microporous polymers: a new organic platform for highly efficient energy storage. Chem. Commun. 50, 4788-4790 (2014).

14. Kou, Y., Xu, Y., Guo, Z. \& Jiang, D. Supercapacitive Energy Storage and Electric Power Supply Using an Aza-Fused $\pi$-Conjugated Microporous Framework. Angew. Chemie Int. Ed. 50, 8753-8757 (2011).

15. Lee, J.-S. M. et al. Porosity-engineered carbons for supercapacitive energy storage using conjugated microporous polymer precursors. J. Mater. Chem. A 4, 7665-7673 (2016).

16. DeBlase, C. R., Silberstein, K. E., Truong, T.-T., Abruña, H. D. \& Dichtel, W. R. $\beta$-Ketoenamine-Linked Covalent Organic Frameworks Capable of Pseudocapacitive Energy Storage. J. Am. Chem. Soc. 135, 1682116824 (2013).

17. Liu, W. et al. A two-dimensional conjugated aromatic polymer via C-C coupling reaction. Nat. Chem. 9, 563570 (2017).

18. Xu, H., Tao, S. \& Jiang, D. Proton conduction in crystalline and porous covalent organic frameworks. Nat. Mater. 15, 722-726 (2016).

19. Zhang, Y. et al. Molecularly soldered covalent organic frameworks for ultrafast precision sieving. Sci. Adv. 7, eabe8706 (2021).

20. Liu, J. et al. Self-standing and flexible covalent organic framework (COF) membranes for molecular separation. Sci. Adv. 6, eabb1110 (2020). 
21. Nguyen, H. L. et al. A Porous Covalent Organic Framework with Voided Square Grid Topology for Atmospheric Water Harvesting. J. Am. Chem. Soc. 142, 2218-2221 (2020).

22. Jiang, J.-X. et al. Conjugated Microporous Poly(aryleneethynylene) Networks. Angew. Chemie Int. Ed. 46, 8574-8578 (2007).

23. Ben, T. \& Qiu, S. Porous aromatic frameworks: Synthesis, structure and functions. CrystEngComm 15, 17-26 (2013).

24. Ben, T. et al. Targeted Synthesis of a Porous Aromatic Framework with High Stability and Exceptionally High Surface Area. Angew. Chemie Int. Ed. 48, 9457-9460 (2009).

25. Das, S., Heasman, P., Ben, T. \& Qiu, S. Porous Organic Materials: Strategic Design and Structure-Function Correlation. Chem. Rev. 117, 1515-1563 (2017).

26. He, T., Geng, K. \& Jiang, D. All sp2 carbon covalent organic frameworks. Trends Chem. 3, 431-444 (2021).

27. Hema, K. et al. Topochemical polymerizations for the solid-state synthesis of organic polymers. Chem. Soc. Rev. (2021). doi:10.1039/d0cs00840k

28. Matsumoto, A. Polymer structure control based on crystal engineering for materials design. Polym. J. 35, 93121 (2003).

29. Guo, Q. H. et al. Single-Crystal Polycationic Polymers Obtained by Single-Crystal-to-Single-Crystal Photopolymerization. J. Am. Chem. Soc. 142, 6180-6187 (2020).

30. Kissel, P., Murray, D. J., Wulftange, W. J., Catalano, V. J. \& King, B. T. A nanoporous two-dimensional polymer by single-crystal-to-single-crystal photopolymerization. Nat. Chem. 6, 774-778 (2014).

31. Kory, M. J. et al. Gram-scale synthesis of two-dimensional polymer crystals and their structure analysis by Xray diffraction. Nat. Chem. 6, 779-784 (2014).

32. Kissel, P. et al. A two-dimensional polymer prepared by organic synthesis. Nat. Chem. 4, 287-291 (2012).

33. Feng, X. \& Schlüter, A. D. Towards Macroscopic Crystalline 2D Polymers. Angew. Chemie Int. Ed. 57, 1374813763 (2018).

34. Bouas-Laurent, H., Castellan, A., Desvergne, J. P. \& Lapouyade, R. Photodimerization of anthracenes in fluid solutions: (Part 2) mechanistic aspects of the photocycloaddition and of the photochemical and thermal cleavage. Chem. Soc. Rev. 30, 248-263 (2001).

35. Beaudoin, D., Maris, T. \& Wuest, J. D. Constructing monocrystalline covalent organic networks by polymerization. Nat. Chem. 5, 830-834 (2013).

36. Ma, T. et al. Single-crystal x-ray diffraction structures of covalent organic frameworks. Science (80-. ). 361, 48-52 (2018).

37. Bouas-Laurent, H., Castellan, A., Desvergne, J. P. \& Lapouyade, R. Photodimerization of anthracenes in fluid solution: Structural aspects. Chem. Soc. Rev. 29, 43-55 (2000).

38. Al-Kaysi, R. O., Müller, A. M. \& Bardeen, C. J. Photochemically driven shape changes of crystalline organic nanorods. J. Am. Chem. Soc. 128, 15938-15939 (2006).

39. Schmidt, G. M. J. Photodimerization in the solid state. Pure Appl. Chem. 27, 647-678 (1971).

40. Enkelmann, V. Single-Crystal-to-Single-Crystal Transformations: The Long Wavelength Tail Irradiation Technique. Mol. Cryst. Liq. Cryst. Sci. Technol. Sect. A. Mol. Cryst. Liq. Cryst. 313, 3 (1998).

41. Brancart, J., Van Damme, J., Du Prez, F. \& Van Assche, G. Thermal dissociation of anthracene photodimers in the condensed state: Kinetic evaluation and complex phase behaviour. Phys. Chem. Chem. Phys. 22, 17306$17313(2020)$. 
42. Leskes, M., Madhu, P. K. \& Vega, S. A broad-banded z-rotation windowed phase-modulated Lee-Goldburg pulse sequence for 1H spectroscopy in solid-state NMR. Chem. Phys. Lett. 447, 370-374 (2007).

43. Brown, S. P., Schnell, I., Brand, J. D., Müllen, K. \& Spiess, H. W. An investigation of $\pi-\pi$ packing in a columnar hexabenzocoronene by fast magic-angle spinning and double-quantum 1H solid-state NMR spectroscopy. J. Am. Chem. Soc. 121, 6712-6718 (1999).

44. Salzillo, T. \& Brillante, A. Commenting on the photoreactions of anthracene derivatives in the solid state. CrystEngComm 21, 3127-3136 (2019).

45. Salzillo, T., Zaccheroni, S., Della Valle, R. G., Venuti, E. \& Brillante, A. Micro raman investigation of the photodimerization reaction of 9-cyanoanthracene in the solid state. J. Phys. Chem. C 118, 9628-9635 (2014).

46. Thommes, M. et al. Physisorption of gases, with special reference to the evaluation of surface area and pore size distribution (IUPAC Technical Report). Pure Appl. Chem. 87, 1051-1069 (2015).

47. Dubbeldam, D., Calero, S., Ellis, D. E. \& Snurr, R. Q. RASPA: molecular simulation software for adsorption and diffusion in flexible nanoporous materials. Mol. Simul. 42, 81-101 (2016).

\section{Acknowledgements}

The high-resolution PXRD data was collected at 11-BM of the Advanced Photon Source at Argonne National Laboratory, which was supported by the U. S. Department of Energy, Office of Science, Office of Basic Energy Sciences, under Contract No. DE-AC02-06CH11357. We are thankful to Prof. Marc Baldo and Dr. Dong-Gwang Ha for help with photoluminescence measurements.

\section{Author Contribution}

C.S. and M.D. designed the experiments. C.S. executed the synthesis and characterization. C.S. and J.O. analyzed the adsorption data. G.S. performed crystallographic data collection and analysis. L.Y. collected and analyzed mass spectrometry data as well as analyzed nuclear magnetic resonance data. All authors contributed to writing of the manuscript and have given approval to the final version of the manuscript.

\section{Competing interests}

The authors declare no competing interests. 